\title{
Fractional iteration of differentiable functions
}

\author{
by M. Kuczma (Katowice)
}

§1. In the present paper we shall be concerned with differentiable solutions $\varphi$ of the functional equation

$$
\varphi^{2}(x)=g(x),
$$

where $g$ is a given function and $\varphi^{2}$ denotes the second functional iterate of $\varphi$. (More generally, for a given function $f$ we denote by $f^{n}$ its $n$ - th iterate: $\left.f^{0}(x)=x, f^{n+1}(x)=f\left[f^{n}(x)\right], n=0,1,2, \ldots\right)$ These investigations have been originated by the following problem of Moszner [4]:

Let $D_{k}^{r}, 1 \leqslant r \leqslant+\infty$, denote the class of all mappings

$$
f: R^{k} \rightarrow R^{k}
$$

that are of class $C^{r}$ in the whole $R^{k}$ and have a positive Jacobian in the whole $R^{k}$. Does there exist, for every $g \in D_{k}^{r}$, a solution $\varphi$ of equation (1) belonging to the class $D_{k}^{r}$ ?

As we shall see, the answer to this question is negative:

§ 2. In the present section we exhibit an example of a function $g \in D_{2}^{\infty}$ for which equation (1) has no solution at all. We start with a lemma ( $\left.{ }^{1}\right)$.

LEMMA 1. Let $E$ be an arbitrary set and $g$ arbitrary function on $E$ taking values in $E$. Further suppose that there exist in $E$ points $a \neq b$ such that $g(a)=b, g(b)=a$, and $g^{2}(x)=x$ implies that either $x=a$, or $x=b$, or $g(x)=x$. Then equation (1) has no solution in $E$.

Proof. Suppose that a function $\varphi$, defined in $E$, satisfies equation (1) in $E$, and put $c=\varphi(a), d=\varphi(b)$. Then $g^{2}(c)=c$ and $g^{2}(d)=d$. If $c=a$, i.e. $\varphi(a)=a$, then $b=g(a)=\varphi^{2}(a)=a$, contrary to the assumption. If $c=b$, i.e. $\varphi(a)=b$, then $\varphi(b)=\varphi^{2}(a)=g(a)=b$ and $a=g(b)=\varphi^{2}(b)$ $=b$, contrary to the assumption. Lastly, $g(c)=c$ implies $\varphi(b)=\varphi[g(a)]$ $=\varphi^{3}(a)=g[\varphi(a)]=g(c)=c$, i.e. $\varphi(a)=\varphi(b)$, whence $g(a)=g(b)$ and $b=a$, again a contradiction.

(1) This lemma is implied by the general considerations of Isaacs [1]. (Cf. also [3], theorem 15.6.) But we sketch here a proof because of its simplicity. 
Now we proceed to give the example announced.

Example I. We consider the transform $g: R^{2} \rightarrow R^{2}$ given by

$$
g:\left\{\begin{array}{l}
x^{\prime}=-\frac{1}{2}\left(x^{2}+y^{2}+1\right)(x+y) \\
y^{\prime}=-\frac{1}{2}\left(x^{2}+y^{2}+1\right) y .
\end{array}\right.
$$

Let us write shortly $u=u(x, y)=\frac{1}{2}\left(x^{2}+y^{2}+1\right)$. Then $u_{x}=x$, $u_{y}=y$ and

$$
\begin{aligned}
\left|\begin{array}{ll}
\frac{\partial x^{\prime}}{\partial x} & \frac{\partial x^{\prime}}{\partial y} \\
\frac{\partial y^{\prime}}{\partial x} & \frac{\partial y^{\prime}}{\partial y}
\end{array}\right| & =\left|\begin{array}{ll}
-x(x+y)-u & -y(x+y)-u \\
-x y & -y^{2}-u
\end{array}\right| \\
& =\left(x^{2}+x y+u\right)\left(y^{2}+u\right)-x y\left(x y+y^{2}+u\right)=u\left(x^{2}+y^{2}+u\right) \geqslant \frac{1}{4}
\end{aligned}
$$

Consequently $g \in D_{2}^{\infty}$. For $a=(1,0)$ and $b=(-1,0)$ we have $g(a)=b$ and $g(b)=a$. The equation $g^{2}(x, y)=(x, y)$ has the form

$$
-u^{\prime}\left(x^{\prime}+y^{\prime}\right)=x, \quad-u^{\prime} y^{\prime}=y,
$$

where

$$
u^{\prime}=u\left(x^{\prime}, y^{\prime}\right)=\frac{1}{2}\left[\left(x^{\prime}\right)^{2}+\left(y^{\prime}\right)^{2}+1\right]=\frac{1}{2}+\frac{1}{2} u^{2}\left(x^{2}+2 x y+2 y^{2}\right) .
$$

Thus (3) may be written as

$$
u^{\prime} u(x+2 y)=x, \quad u^{\prime} u y=y .
$$

If $u^{\prime} u \neq 1$, then from the second equation of (4) we obtain $y=0$, and if $u^{\prime} u=1$, then $y=0$ results from the first equation of (4). Consequently $y$ must be zero, $u^{\prime} u$ reduces to

$$
u^{\prime} u=\left(\frac{1}{2}+\frac{x^{2}}{2}\left(\frac{x^{2}+1}{2}\right)^{2}\right) \frac{x^{2}+1}{2}=\frac{1}{16}\left(4+x^{2}\left(x^{2}+1\right)^{2}\right)\left(x^{2}+1\right)
$$

and system (4) to

$$
u^{\prime} u x=x
$$

If $u^{\prime} u \neq 1$, then $x=0$, and consequently we get the solution $(x, y)$ $=(0,0)$, which is a fixed point of $g: g(0,0)=(0,0)$. To solve the equation $u^{\prime} u=1$ we put $t=x^{2}+1$ and thus the equation becomes

i.e.

$$
t^{4}-t^{3}+4 t-16=0,
$$

$$
(t-2)\left(t^{3}+t^{2}+2 t+8\right)=0 .
$$

But, since $t=x^{2}+1 \geqslant 1$, we have $t^{3}+t^{2}+2 t+8 \geqslant 12$ and consequently $t=2$ is the only admissible solution of (5). It leads us to $x=1$ 
and $x=-1$, i.e. $a=(1,0)$ and $b=(-1,0)$ are the only fixed points of $g^{2}$ that are not fixed points of $g$.

As we see, the function $g$ given by (2) fulfils the assumptions of Lemma 1, and thus equation (1) has no solution in $R^{2}$.

§ 3. A similar situation cannot happen if $k=1$. Equation (1) with a continuous and strictly increasing function $g$ on $(-\infty,+\infty)$ always has a continuous and strictly increasing solution $\varphi$ (cf. [3], corollary to theorem 15.7). In particular, we have the following

LEMMA 2 ([2], [3]). Let $g(x)$ be continuous and strictly increasing in an interval $(a, b),-\infty \leqslant a<b \leqslant+\infty$, moreover, let $a<g(x)<x$ in $(a, b)$.

Further let $x_{0}, y_{0}$ be arbitrary two points of $(a, b)$ such that $g\left(x_{0}\right)$ $<y_{0}<x_{0}$, and let $\varphi_{0}(x)$ be an arbitrary continuous and strictly increasing function on $\left\langle y_{0}, x_{0}\right\rangle$ such that

$$
\varphi_{0}\left(x_{0}\right)=y_{0}, \quad \varphi_{0}\left(y_{0}\right)=g\left(x_{0}\right)
$$

Then there exists a unique continuous and strictly increasing function $\varphi(x)$ on $(a, b)$ satisfying equation $(1)$ in $(a, b)$ and such that

$$
\varphi(x)=\varphi_{0}(x) \quad \text { for } x \in\left\langle y_{0}, ;_{i 0}^{*}\right\rangle
$$

As an immediate consequence of Lemma 2 we obtain the following

LEMma 3. If the function $g(x)$ fulfils the conditions of Lemma 2, then every continuous and strictly increasing solution $\varphi(x)$ of equation (1) in $(a, b)$ is completely determined by its values in an arbitrary right neighbourhood $(a, a+\delta)$ of the point $a$.

Now we shall prove an analogue of Lemma 2 for differentiable functions.

THEOREM 1. Under conditions of Lemma 2, if, moreover, $g(x)$ is of class $C^{1}$ in $(a, b)$ with $g^{\prime}(x)>0$ in $(a, b)$, and $\varphi_{0}(x)$ is of class $C^{1}$ in $\left\langle y_{0}, x_{0}\right\rangle$ with $\varphi_{0}^{\prime}(x)>0$ in $\left\langle y_{0}, x_{0}\right\rangle$, and if $\left(^{2}\right)$

$$
\varphi_{0}^{\prime}\left(y_{0}\right)=\frac{g^{\prime}\left(x_{0}\right)}{\varphi_{0}^{\prime}\left(x_{0}\right)}
$$

then the continuous and strictly increasing solution $\varphi(x)$ of equation (1) fulfilling (7) is of class $C^{1}$ in $(a, b)$.

(2) $\varphi_{0}^{\prime}\left(x_{0}\right)$ and $\varphi_{0}^{\prime}\left(y_{0}\right)$ denote here the left derivative and the right derivative of $\varphi_{0}$ at the points $x_{0}$ and $y_{0}$, respectively. 
Proof. Let the sequence $x_{n}$ be defined for integral $n\left({ }^{3}\right)$ by

$$
x_{n}=\varphi^{n}\left(x_{0}\right) \text {. }
$$

It is readily seen that the sequence $x_{n}$ is strictly decreasing and

$$
(a, b)=\bigcup_{n=n_{0}}^{\infty}\left\langle x_{n+1}, x_{n}\right)
$$

where $n_{0}=-\infty$ if $\lim _{x \rightarrow b-0} g(x)=b$, and if $\lim _{x \rightarrow b-0} g(x)=b^{\prime}<b$, then $n_{0}$ is determined by the condition $x_{n_{0}+1} \epsilon\left\langle b^{\prime}, b\right)$ and we put $x_{n_{0}} \stackrel{\text { df }}{=} b$. At any case $n_{0}<0$.

By (6) and (9)

$$
\varphi_{0}^{-1}(x) \epsilon\left(y_{0}, x_{0}\right) \quad \text { for } x \epsilon\left(x_{2}, x_{1}\right)=\left(g\left(x_{0}\right), y_{0}\right) \text {. }
$$

In virtue of (1) and (7) we get hence for $x \in\left(g\left(x_{0}\right), y_{0}\right)$

$$
\varphi(x)=\varphi\left(\varphi_{0}\left(\varphi_{0}^{-1}(x)\right)\right)=\varphi\left(\varphi\left(\varphi_{0}^{-1}(x)\right)\right)=g\left[\varphi_{0}^{-1}(x)\right],
$$

which shows that $\varphi$ is of class $C^{1}$ in $\left(g\left(x_{0}\right), y_{0}\right)=\left(x_{2}, x_{1}\right)$. Now

$$
\lim _{x \rightarrow y_{0}+0} \varphi^{\prime}(x)=\lim _{x \rightarrow y_{0}+0} \varphi_{0}^{\prime}(x)=\varphi_{0}^{\prime}\left(y_{0}\right),
$$

since $\varphi_{0}$ is of class $C^{1}$ in $\left\langle y_{0}, x_{0}\right\rangle$, and

$$
\lim _{x \rightarrow y_{0}-0} \varphi^{\prime}(x)=\lim _{x \rightarrow x_{0}-0} \varphi^{\prime}[\varphi(x)]=\lim _{x \rightarrow x_{0}-0} \frac{g^{\prime}(x)}{\varphi^{\prime}(x)}=\lim _{x \rightarrow x_{0}-0} \frac{g^{\prime}(x)}{\varphi_{0}^{\prime}(x)}=\frac{g^{\prime}\left(x_{0}\right)}{\varphi_{0}^{\prime}\left(x_{0}\right)}=\varphi_{0}^{\prime}\left(y_{0}\right),
$$

since $\varphi$ satisfies equation (1) and $\varphi_{0}$ is of class $C^{1}$ in $\left\langle y_{0}, x_{0}\right\rangle$ and fulfils (8). This proves, in view of the continuity of $\varphi$, that $\varphi^{\prime}$ exists and is continuous at $x=x_{0}$. Consequently $\varphi$ is of class $C^{1}$ in $\left(x_{2}, x_{0}\right)$.

Now suppose that $\varphi$ is of class $C^{1}$ in $\left(x_{n}, x_{0}\right)$ for an $n \geqslant 2$. According to (9) $\varphi\left(x_{i}\right)=x_{i+1}$ for all $i>n_{0}$, and consequently

$$
\varphi^{-1}(x) \in\left(x_{n}, x_{n-2}\right) \quad \text { for } x \in\left(x_{n+1}, x_{n-1}\right) \text {. }
$$

Furthermore, $\varphi^{-1}$ is of class $C^{1}$ in $\left(x_{n+1}, x_{n-1}\right)$, since differentiating (1) we get $\varphi^{\prime}(x) \neq 0$ for $x \in\left(x_{n}, x_{n-2}\right)$. Thus, for $x \in\left(x_{n+1}, x_{n-1}\right)$,

$$
\varphi(x)=\varphi\left(\varphi\left(\varphi^{-1}(x)\right)\right)=g\left[\varphi^{-1}(x)\right]
$$

is of class $C^{1}$, and consequently $\varphi$ is of class $C^{1}$ in $\left(x_{n+1}, x_{0}\right)$. Induction now yields that $\varphi$ is of class $C^{1}$ in $\left(x_{n+1}, x_{0}\right)$ for every $n \geqslant 0$, and quite similarly one can prove that $\varphi$ is of class $C^{1}$ in $\left(x_{1}, x_{-n}\right)$ for every $n \leqslant-n_{0}$. By (10) $\varphi$ is of class $C^{1}$ in $(a, b)$, which was to be proved. to $\varphi^{-n}$.

(') Positive and negative. For negative $n, \varphi^{n}$ denotes the inverse function 
The following lemma is easily established by induction.

LEMMA 4. If a function $\varphi$ is of class $C^{r}$ in $(a, b)$ and takes values in $(a, b)$, then

$$
\frac{d^{j}}{d x^{j}} \varphi^{2}(x)=\sum_{i=1}^{j} P_{i j}\left(\varphi^{\prime}(x), \ldots, \varphi^{(j)}(x)\right) \varphi^{(i)}[\varphi(x)], \quad j=1, \ldots, r,
$$

where $P_{i j}\left(t_{1}, \ldots, t_{j}\right)$ are polynomials.

By almost the same argument as in the proof of Theorem 1 one can prove the following

THEOREM 2. Under conditions of Lemma 2 , if, moreover, $g(x)$ is of class $C^{r}$ in $(a, b), 1 \leqslant r \leqslant \infty$, with $g^{\prime}(x)>0$ in $(a, b)$, and $\varphi_{0}(x)$ is of class $C^{r}$ in $\left\langle y_{0}, x_{0}\right\rangle$ with $\varphi_{0}^{\prime}(x)>0$ in $\left\langle y_{0}, x_{0}\right\rangle$, and if $\left.{ }^{4}\right)$

$$
\sum_{i=1}^{j} P_{i j}\left(\varphi_{0}^{\prime}\left(x_{0}\right), \ldots, \varphi_{0}^{(j)}\left(x_{0}\right)\right) \varphi_{0}^{(i)}\left(y_{0}\right)=g^{(j)}\left(x_{0}\right), \quad j=1, \ldots, r,
$$

where $P_{i j}$ are the polynomials occurring in Lemma 4 , then the continuous and strictly increasing solution $\varphi(x)$ of equation (1) fulfilling (7) is of class $C^{r}$ in $(a, b)$.

The following result is now an easy consequence of Theorem 2 .

THEOREM 3. If $g \in D_{1}^{r}, 1 \leqslant r \leqslant \infty$, and $g(x) \neq x$ in $(-\infty,+\infty)$, then equation (1) has a solution $\varphi \in D_{1}^{r}$.

Proof. The condition $g(x) \neq x$ implies that either $g(x)<x$ in $(-\infty,+\infty)$, or $g(x)>x$ in $(-\infty,+\infty)$. In the former case Theorem 2 with $(a, b)=(-\infty,+\infty)$ shows that equation (1) has a strictly increasing solution $\varphi$ of class $C^{r}$ in $(-\infty,+\infty)$. Since $\varphi^{\prime}[\varphi(x)] \varphi^{\prime}(x)=g^{\prime}(x) \neq 0$, $\varphi$ must belong to $D_{1}^{r}$.

If $g(x)>x$ in $(-\infty,+\infty)$, then $f(x)=-g(-x)<x$ in $(-\infty,+\infty)$ and $f$ belongs to $D_{1}^{r}$. Thus there exists a $\psi \in D_{1}^{r}$ such that $\psi^{2}(x)=f(x)$ in $(-\infty,+\infty)$. The function $\varphi(x)=-\psi(-x)$ belongs to $D_{1}^{r}$ and satisfies equation (1) in $(-\infty,+\infty)$.

§ 4. The conclusion of Theorem 3 fails to hold if $g$ has fixed points in $(-\infty,+\infty)$, as may be seen from examples IV and $\mathrm{V}$ in $\S 5$. In the present section we shall consider equation (1) in an interval $\langle a, b)$, $-\infty<a<b \leqslant+\infty$, under the condition that $a<g(x)<x$ in $(a, b)$, $g^{\prime}(x)$ exists, is continuous and positive in $\langle a, b)$, in particular $\left(^{5}\right)$

$$
g^{\prime}(a)=s>0 .
$$

(4) The derivatives of $\varphi_{0}$ at $x_{0}$ and $y_{0}$ denote the left and right derivatives, respectively.

(s) $g^{\prime}(a)$ denotes the right derivative of $g$ at $a$. 
Suppose that equation (1) has a strictly increasing solution $\varphi$ that is of class $C^{\mathbf{l}}$ in $\langle a, b)$. Differentiating (1) we obtain

$$
\varphi^{\prime}[\varphi(x)] \varphi^{\prime}(x)=g^{\prime}(x) \text {. }
$$

The function $\varphi(x)$ fulfils the condition (cf. [5])

$$
g(x)<\varphi(x)<x \quad \text { in }(a, b),
$$

whence it follows in view of the continuity of $\varphi$ and $g$ at $a$ that

$$
\varphi(a)=a .
$$

Thus we obtain by (12) and (11)

$$
\varphi^{\prime}(a)=\sqrt{s} .
$$

Replacing in (12) $x$ by $\varphi(x)$ we get in view of (1)

$$
\varphi^{\prime}[g(x)] \varphi^{\prime}[\varphi(x)]=g^{\prime}[\varphi(x)],
$$

whence by (12)

$$
\frac{\varphi^{\prime}(x)}{\varphi^{\prime}[g(x)]}=\frac{g^{\prime}(x)}{g^{\prime}[\varphi(x)]}
$$

By (1) $\varphi\left[g^{i}(x)\right]=\varphi^{2 i+1}(x)=g^{i}[\varphi(x)]$. Consequently we obtain by (15)

whence

$$
\frac{\varphi^{\prime}\left[g^{i}(x)\right]}{\varphi^{\prime}\left[g^{i+1}(x)\right]}=\frac{g^{\prime}\left[g^{i}(x)\right]}{g^{\prime}\left[g^{i}(\varphi(x))\right]}, \quad i=0,1,2, \ldots,
$$

$$
\frac{\varphi^{\prime}(x)}{\varphi^{\prime}\left[g^{n}(x)\right]}=\prod_{i=0}^{n-1} \frac{g^{\prime}\left[g^{i}(x)\right]}{g^{\prime}\left[g^{i}(\varphi(x))\right]}, \quad n==1,2,3, \ldots
$$

As $n \rightarrow \infty$, we obtain from (16) and (14) for $x \in(a, b)$

$$
\varphi^{\prime}(x)=\sqrt{s} \prod_{i=0}^{\infty} \frac{g^{\prime}\left[g^{i}(x)\right]}{g^{\prime}\left[g^{i}(\varphi(x))\right]}
$$

(17) shows that for $y=\varphi(x)$ the infinite product

$$
G(x, y)=\prod_{i=0}^{\infty} \frac{g^{\prime}\left[g^{i}(x)\right]}{g^{\prime}\left[g^{i}(y)\right]}
$$

must converge and the function $\varphi$ satisfies in $(a, b)$ the differential equation

$$
\varphi^{\prime}=\sqrt{s} G(x, \varphi)
$$


Thus we have proved the following

THEOREM 4. If $g(x)$ is of class $C^{1}$ in $\langle a, b), a<g(x)<x$ in $(a, b)$ and $g^{\prime}(x)>0$ in $\langle a, b)$, and if $\varphi(x)$ is a strictly increasing solution of equation (1) in $\langle a, b)$, of class $C^{1}$ in $\langle a, b)$, then $\varphi$ satisfies in $(a, b)$ the differential equation (19), where $s$ and $G$ are given by (11) and (18), respectively.

A more detailed discussion of equation (19) as well as of problems of the uniqueness and existence connected therewith is deferred to a future publication.

§ 5. We conclude the paper with a few examples.

EXAMPLE II. Let $g(x)=s(x--a)+a, \quad 0<s<1, \quad x \in\langle a, b)$. Then $g^{\prime}(x) \equiv s$ and $G(x, y) \equiv 1$. Equation (19) becomes $\varphi^{\prime}=\sqrt{s}$ which together with (13) implies that $\varphi(x)=\sqrt{s}(x-a)+a$.

If we have $g(x)=s(x-a)+a$ in an interval $(c, a\rangle,-\infty \leqslant c<a$ $<+\infty$, and $\varphi$ is a strictly increasing solution of equation (1), of class $C^{1}$ in $(c, a)$, then the function $\psi(x)=2 a-\varphi(2 a-x)$ is a strictly increasing solution of the equation

$$
\psi^{2}(x)=2 a-g(2 a-x)
$$

of class $C^{1}$ in the interval $\langle a, 2 a-c)$. But, since $2 a-g(2 a-x)=s(x-a)+a$, we must have $\psi(x)=\sqrt{s}(x-a)+a$ in $\langle a, 2 a-c)$, whence also $\varphi(x)$ $=\sqrt{s}(x-a)+a$ in $(c, a)$.

If $s>1$ and $\varphi(x)$ is a solution of equation (1) with $g(x)=s(x-a)+a$, of class $C^{1}$ in an interval $I$ containing $a$, then $\psi(x)=\varphi^{-1}(x)$ is a solution of the equation

$$
\psi^{2}(x)=s^{-1}(x-a)+a,
$$

of class $C^{1}$ in $\varphi(I)$. Consequently $\psi(x)=(\sqrt{s})^{-1}(x-a)+a$ in $\varphi(I)$ and $\varphi(x)=\sqrt{s}(x-a)+a$ in $I$.

If $s=1$, then $g(x)=x$ and $\varphi(x)=x$ is the only increasing solution of equation (1) in any interval $I$ ([3], theorem 15.2).

Thus we arrive at the following conclusion:

THEOREM 5. If

$$
g(x)=s(x-a)+a, \quad s>0,
$$

in an interval $I \subset(-\infty,+\infty)$, where $a \in I$, then

$$
\varphi(x)=\sqrt{s}(x-a)+a
$$

is the only strictly increasing solution of equation (1) of class $C^{\mathbf{1}}$ in $I$. 
EXAMPLE III. Let $\langle a, b)=\langle 0, \infty), \quad g(x)=x /(x+1)$. Then $g^{\prime}(x)$ $=1 /(x+1)^{2}, g^{n}(x)=x /(n x+1), s=1$, and

$$
G(x, y)=\lim _{n \rightarrow \infty} \prod_{i=0}^{n-1} \frac{\left(1+\frac{y}{i y+1}\right)^{2}}{\left(1+\frac{x}{i x+1}\right)^{2}}=\lim _{n \rightarrow \infty} \frac{(n y+1)^{2}}{(n x+1)^{2}}=\frac{y^{2}}{x^{2}} .
$$

Equation (19) becomes

$$
\varphi^{\prime}=[\varphi]^{2} / x^{2}
$$

and has the solutions $\varphi(x) \equiv 0$ and $\varphi(x)=x /(c x+1)$. The former evidently does not satisfy (1) and inserting the latter into (1) we see that $c$ must be equal to $\frac{1}{2}$. Thus finally we get

$$
\varphi(x)=\frac{x}{\frac{1}{2} x+1} .
$$

Note that in this case not every solution of equation (19) satisfies equation (1).

EXAMPLE IV. Let us fix arbitrary $s, s^{\prime}$ such that

and put

$$
\mathbf{0}<s<\mathbf{1}<s^{\prime},
$$

Then

$$
u=\frac{1}{2} \frac{s^{\prime}-1}{s^{\prime}-\sqrt{s}}, \quad v=1-\frac{1-\sqrt{s} u}{s^{\prime}}
$$

$$
v-u=\frac{1}{s^{\prime}}\left[\left(s^{\prime}-1\right)-\left(s^{\prime}-\sqrt{s}\right) u\right]=\frac{s^{\prime}-1}{2 s^{\prime}}>0,
$$

and thus

$$
0<u<v<1 \text {. }
$$

Let $h(x)$ be an arbitrary (but fixed) convex function, of class $C^{1}$ in $\langle u, v\rangle$, and fulfilling the following conditions:

$$
h(u)=s u, \quad h(v)=\sqrt{s} u, \quad h^{\prime}(u)=s, \quad h^{\prime}(v)=s^{\prime} .
$$

Such a function surely exists, since

$$
\begin{aligned}
h(v)-h(u) & =(\sqrt{s}-s) u=\frac{1}{2}\left(s^{\prime}-1\right) \frac{\sqrt{s}-s}{s^{\prime}-\sqrt{s}} \\
& <\frac{1}{2}\left(s^{\prime}-1\right) \frac{\sqrt{s}-s}{1-\sqrt{s}}<\frac{s^{\prime}-1}{2}=s^{\prime}(v-u) .
\end{aligned}
$$

We define the function $g(x)$ in $(-\infty,+\infty)$ as follows (cf. Fig. 1):

$$
g(x)= \begin{cases}s x & \text { for } x \in(-\infty, u), \\ h(x) & \text { for } x \in\langle u, v\rangle, \\ s^{\prime}(x-1)+1 & \text { for } x \in(v,+\infty)\end{cases}
$$


The function $g$ thus defined belongs to the class $D_{1}^{1}$, and if we choose $h(x)$ more thoroughly we may make $g$ to belong to $D_{1}^{r}$ with any $r \geqslant 1$. Moreover, let us note that, since $h$ is convex on $\langle u, v\rangle$, we have

$$
s \leqslant g^{\prime}(x) \leqslant s^{\prime} .
$$

We shall show that equation (1) with $g$ defined by (20) has not a solution $\varphi \in D_{1}^{1}$. Supposing the contrary, let $\varphi$ be such a solution. By Theorem 5

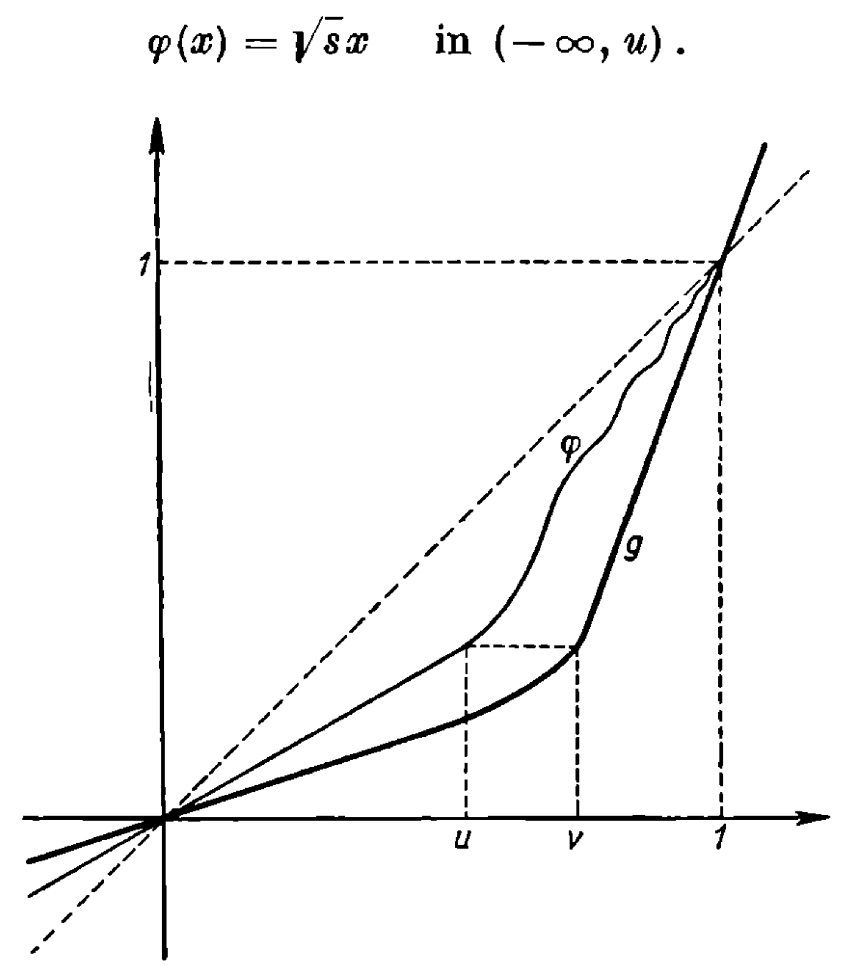

Fig. 1

In virtue of Lemma 3 the function $\varphi(x)$ is completely determined by $(22)$ in $(0,1)$. But it has been proved in [5] that $\lim _{x \rightarrow 1-0} \varphi^{\prime}(x)$ does not exist. Consequently $\varphi$ cannot be of class $C^{1}$ in $(-\infty,+\infty)$.

In the above example, though equation (1) has no solution of class $C^{1}$ in the whole $(-\infty,+\infty)\left({ }^{6}\right)$, nevertheless every point of $(-\infty,+\infty)$ has a neighbourhood in which equation (1) has a local solution of class $C^{1}$. (The function indicated on Fig. 1 is of class $C^{1}$ in $(-\infty, 1)$, and a similar construction can be carried out in the interval $(0,+\infty)$.) But we can

$\left.{ }^{(}{ }^{8}\right)$ The argument presented shows that equation (1) has no strictly increasing solution of class $C^{1}$ in $(-\infty,+\infty)$; but every solution of (1) must be invertible ([3], lemma 15.1) and (1) cannot have strictly decreasing solutions, since function (20) has an éren number of fixed points ([3], theorem 15.10). 
modify this example as to obtain an equation that has not eren a local solution of class $C^{1}$ in a neighbourhood of zero.

EXAMPLE V. Function (20) occurring in example IV depends on $s$ and $s^{\prime}$. (For given $s$ and $s^{\prime}$ we consider the function $h(x)$ as fixed.) To make this dependence more explicit we shall denote function (20) restricted to the interval $\langle 0,1\rangle$ by $g^{*}\left(s, s^{\prime} ; x\right)$.

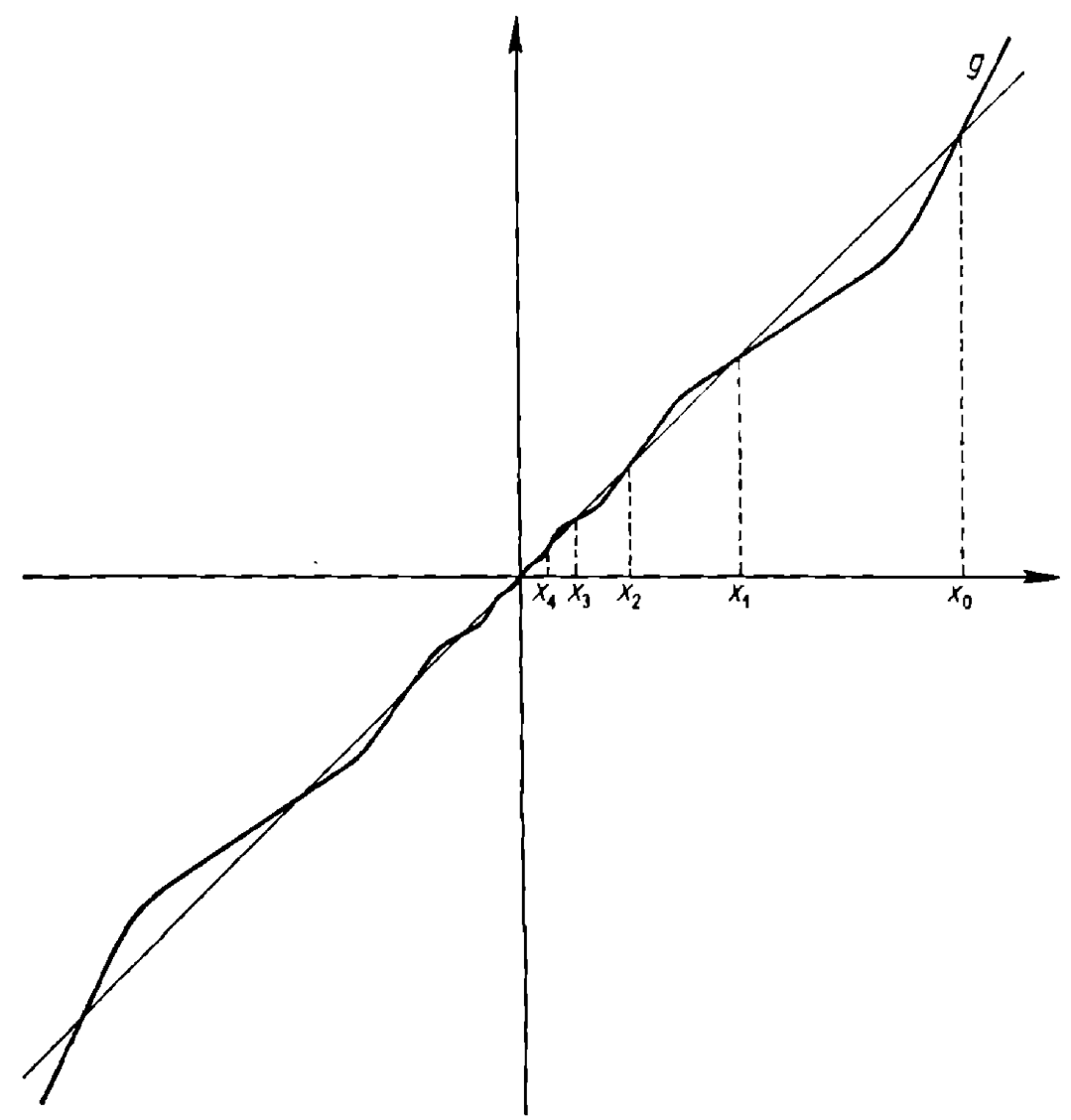

Fig. 2

We put $x_{n}=2^{-n}, s_{n}=2^{-\left(2^{-n}\right)}, n=0, \pm 1, \pm 2, \ldots$ These sequences are strictly monotonic and

$$
\begin{array}{ll}
\lim _{n \rightarrow+\infty} x_{n}=0, & \lim _{n \rightarrow-\infty} x_{n}=+\infty, \\
\lim _{n \rightarrow+\infty} s_{n}=1, & \lim _{n \rightarrow-\infty} s_{n}=0 .
\end{array}
$$

For every $n$ we define the function $g_{n}(x)$ on $\left\langle x_{n+1}, x_{n}\right\rangle$ by

$$
g_{n}(x)=x_{n+1}+\left(x_{n}-x_{n+1}\right) g^{*}\left(s_{n+1}, s_{n}^{-1} ; \frac{x-x_{n+1}}{x_{n}-x_{n+1}}\right) \text {. }
$$

In view of (21) we have

$$
s_{n+1} \leqslant g_{n}^{\prime}(x) \leqslant s_{n}^{-1} \quad \text { for } x \epsilon\left\langle x_{n+1}, x_{n}\right\rangle \text {. }
$$


Now we define the function $g(x)$ on $\langle 0,+\infty)$ as follows:

$$
g(x)= \begin{cases}g_{n}(x) & \text { for } x \in\left(x_{n+1}, x_{n}\right\rangle, n=0, \pm 2, \pm 4, \ldots, \\ g_{n}^{-1}(x) & \text { for } x \in\left(x_{n+1}, x_{n}\right\rangle, n= \pm 1, \pm 3, \ldots, \\ 0 & \text { for } x=0,\end{cases}
$$

and we extend $g(x)$ onto $(-\infty,+\infty)$ by the condition that it is odd:

$$
g(x)=-g(-x)
$$

(ef. Fig. 2). Function (24) clearly is of class $C^{1}$ in $(-\infty, 0) \cup(0,+\infty)$. But condition (23) together with (25) show that

$$
\lim _{n \rightarrow \infty} g^{\prime}(x)=1 \text {. }
$$

Since $g(x)$ is continuous, condition (26) implies that $g^{\prime}(0)$ exists and equals 1. Thus $g$ is of class $C^{1}$ in $(-\infty,+\infty)$ and in fact $g \in D_{1}^{1}$ (cf. in particular (23) and (25)).

It follows from what has been shown in example IV that equation (1) with the function $g$ given by (24) cannot have a strictly increasing solution $\varphi$ of class $C^{1}$ in $\left\langle x_{n+1}, x_{n}\right\rangle$ (for any $n$ ). Consequently equation (1) cannot have a.strictly increasing solution of class $C^{1}$ in any neighbourhood of the origin.

\section{References}

[1] R. Isaacs, Iterates of fractional order, Canadian J. Math. 2 (1950), pp. 409-416.

[2] M. Kuczma, On the functional equation $\varphi^{n}(x)=g(x)$, Ann. Polon. Math. 11 (1961), pp. 161-175.

[3] - Functional equations in a single variable, Warszawa 1968.

[4] Z. Moszner, Problème P.2, Aequationes Math. 1 (1968), p. 150.

[5] J. Wójcik-Ger, On convex solutions of the functional equation $\varphi^{2}(x)=g(x)$, Zeszyty Naukowe Uniw. Jagiell., Prace Mat. (to appear). 